PROCEEDINGS OF THE

AMERICAN MATHEMATICAL SOCIETY

Volume 128, Number 1, Pages 203-213

S 0002-9939(99)05012-1

Article electronically published on June 21, 1999

\title{
RADEMACHER AND GAUSSIAN AVERAGES AND RADEMACHER COTYPE OF OPERATORS BETWEEN BANACH SPACES
}

\author{
AICKE HINRICHS
}

(Communicated by Dale Alspach)

\begin{abstract}
A basic result of B. Maurey and G. Pisier states that Gaussian and Rademacher averages in a Banach space $X$ are equivalent if and only if $X$ has finite cotype. We complement this for linear bounded operators between Banach spaces. For $T \in \mathcal{L}(X, Y)$, let $\varrho\left(T \mid \mathcal{G}_{n}, \mathcal{R}_{n}\right)$ be the least $c$ such that

$$
\left(\mathbf{E}\left\|\sum_{k=1}^{n} T x_{k} g_{k}\right\|^{2}\right)^{1 / 2} \leq c\left(\mathbf{E}\left\|\sum_{k=1}^{n} x_{k} r_{k}\right\|^{2}\right)^{1 / 2}
$$

where $\mathcal{G}_{n}=\left(g_{1}, \ldots, g_{n}\right)$ and $\mathcal{R}_{n}=\left(r_{1}, \ldots, r_{n}\right)$ are systems of $n$ independent standard Gaussian and Rademacher variables, respectively. Let $\varrho\left(T \mid \mathcal{I}_{n}, \mathcal{R}_{n}\right)$ be the Rademacher cotype 2 norm of $T$ computed with $n$ vectors. We prove inequalities showing that the asymptotic behaviour of the sequence $\varrho\left(T \mid \mathcal{G}_{n}, \mathcal{R}_{n}\right)$ is almost determined by the asymptotic behaviour of the sequence $\varrho\left(T \mid \mathcal{I}_{n}, \mathcal{R}_{n}\right)$. In particular, we get
\end{abstract}

$$
\varrho\left(T \mid \mathcal{G}_{n}, \mathcal{R}_{n}\right)=o(\sqrt{1+\log n}) \text { if and only if } \varrho\left(T \mid \mathcal{I}_{n}, \mathcal{R}_{n}\right)=o(\sqrt{n}) .
$$

\section{INTRODUCTION}

This paper is motivated by a classical result of B. Maurey and G. Pisier [4] stating the equivalence of Gaussian and Rademacher averages in Banach spaces of finite cotype. We investigate in which sense and to what extent this remains true in the case of linear and bounded operators between Banach spaces.

We use standard Banach space notation. Throughout this paper, $X$ and $Y$ are Banach spaces. By operator we always mean a linear and bounded operator between Banach spaces. $\mathcal{L}(X, Y)$ denotes the Banach space of all operators from $X$ into $Y$. $T$ will always be an operator from $X$ into $Y$. Since we will need a couple of ideal norms arising from averages with respect to different orthonormal systems, let us introduce some convenient general notation adopted from the monograph [5]. Given two orthonormal systems of $n$ functions $\mathcal{A}_{n}=\left(a_{1}, \ldots, a_{n}\right)$ and $\mathcal{B}_{n}=\left(b_{1}, \ldots, b_{n}\right)$ in Hilbert spaces $L_{2}(M, \mu)$ and $L_{2}(N, \nu)$, respectively, we define $\varrho\left(T \mid \mathcal{B}_{n}, \mathcal{A}_{n}\right)$ as the

Received by the editors June 5, 1997 and, in revised form, March 18, 1998.

1991 Mathematics Subject Classification. Primary 47D50, 46B07.

The author is supported by DFG grant PI 322/1-1. The content of this paper is part of the author's PhD-thesis written under the supervision of A. Pietsch.

(C)1999 American Mathematical Society 
least constant $c$ such that

$$
\left(\int_{N}\left\|\sum_{k=1}^{n} b_{k}(t) T x_{k}\right\|^{2} d \nu(t)\right)^{1 / 2} \leq c\left(\int_{M}\left\|\sum_{k=1}^{n} a_{k}(s) x_{k}\right\|^{2} d \mu(s)\right)^{1 / 2}
$$

whenever $x_{1}, \ldots, x_{n} \in X$. We write $\varrho\left(X \mid \mathcal{B}_{n}, \mathcal{A}_{n}\right)$ for $\varrho\left(I_{X} \mid \mathcal{B}_{n}, \mathcal{A}_{n}\right)$, where $I_{X}$ is the identity of $X$. Let $\mathcal{I}_{n}$ be the unit vector basis in $l_{2}^{n}$. Let $\mathcal{G}_{n}=\left(g_{1}, \ldots, g_{n}\right)$ and $\mathcal{R}_{n}=\left(r_{1}, \ldots, r_{n}\right)$ be systems of $n$ i.i.d. Rademacher and standard Gaussian random variables, respectively. Then $\varrho\left(T \mid \mathcal{I}_{n}, \mathcal{R}_{n}\right)$ is the Rademacher cotype 2 norm of $T$ computed with $n$ vectors. We use the notation $\varrho_{2}^{(q)}\left(T \mid \mathcal{I}_{n}, \mathcal{R}_{n}\right)$ for the Rademacher cotype $q$ norm of $T$ computed with $n$ vectors, that is, the least $c$ such that

$$
\left(\sum_{k=1}^{n}\left\|T x_{k}\right\|^{q}\right)^{1 / q} \leq c\left(\mathbf{E}\left\|\sum_{k=1}^{n} x_{k} r_{k}\right\|^{2}\right)^{1 / 2}
$$

for all $x_{1}, \ldots, x_{n} \in X$.

It is well-known that the sequence $\varrho\left(X \mid \mathcal{R}_{n}, \mathcal{G}_{n}\right)$ is bounded for any Banach space $X$ by a universal constant; see [4]. The mentioned result of B. Maurey and G. Pisier [4] is the following dichotomy. For a Banach space $X$, either $\varrho\left(X \mid \mathcal{G}_{n}, \mathcal{R}_{n}\right)$ is bounded or $\varrho\left(X \mid \mathcal{G}_{n}, \mathcal{R}_{n}\right) \asymp \sqrt{1+\log n}$. The first case occurs iff $X$ has finite cotype, which means that the sequence $\varrho_{2}^{(q)}\left(T \mid \mathcal{I}_{n}, \mathcal{R}_{n}\right)$ is bounded for some $q \geq 2$. In the second case $X$ has to contain the spaces $l_{\infty}^{n}$ uniformly, or, equivalently, $\varrho\left(T \mid \mathcal{I}_{n}, \mathcal{R}_{n}\right)=\sqrt{n}$ for all $n$. Such a dichotomy is not true for operators. There are examples of diagonal operators in $l_{\infty}$ for which $\varrho\left(T \mid \mathcal{G}_{n}, \mathcal{R}_{n}\right) \asymp(1+\log n)^{\alpha}$ for given $0<\alpha<1 / 2$; see also the examples given in section 4 . Nevertheless, the gap between Gaussian and Rademacher averages in terms of the sequence $\varrho\left(T \mid \mathcal{G}_{n}, \mathcal{R}_{n}\right)$ is closely related to the behaviour of the Rademacher cotype norms of $T$. In section 3 , we show that the behaviour of the sequence $\varrho\left(T \mid \mathcal{G}_{n}, \mathcal{R}_{n}\right)$ for $n \rightarrow \infty$ is almost determined by the Rademacher cotype 2 norms.

\section{BASIC FACTS ON IDEAL NORMS}

An ideal norm $\alpha$ assigns to any operator $T$ a nonnegative number $\alpha(T)$ such that $\alpha$ is a norm on each component $\mathcal{L}(X, Y)$ and satisfies the ideal norm property

$$
\alpha(B T A) \leq\|B\| \alpha(T)\|A\| \text { for } A \in \mathcal{L}\left(X_{0}, X\right), T \in \mathcal{L}(X, Y) \text { and } B \in \mathcal{L}\left(Y, Y_{0}\right) .
$$

We will simply write $\alpha(X)$ for $\alpha\left(I_{X}\right)$. For a systematic treatment of ideal norms and, in particular, for the facts quoted in this section, we refer the reader to [5]. The maps

$$
\varrho\left(\mathcal{B}_{n}, \mathcal{A}_{n}\right): T \longmapsto \varrho\left(T \mid \mathcal{B}_{n}, \mathcal{A}_{n}\right)
$$

with orthonormal systems $\mathcal{A}_{n}$ and $\mathcal{B}_{n}$ defined in the introduction are examples of ideal norms. The index $n$ on an orthonormal system always denotes the cardinality of the system. To write (1.1) in a more comprehensive way, we use the abbreviation

$$
\left\|\left(x_{k}\right) \mid \mathcal{A}_{n}\right\|=\left(\int_{M}\left\|\sum_{k=1}^{n} a_{k}(s) x_{k}\right\|^{2} d \mu(s)\right)^{1 / 2}
$$


for an orthonormal system $\mathcal{A}_{n}=\left(a_{1}, \ldots, a_{n}\right) \subset L_{2}(M, \mu)$ and $x_{1}, \ldots, x_{n} \in X$. Then (1.1) reads as follows:

$$
\left\|\left(T x_{k}\right)\left|\mathcal{B}_{n}\|\leq c\|\left(x_{k}\right)\right| \mathcal{A}_{n}\right\| .
$$

Given ideal norms $\alpha, \beta$ and $\gamma$, we write $\alpha \leq \beta \circ \gamma$ if $\alpha(S T) \leq \beta(S) \gamma(T)$ for any Banach spaces $X, Y, Z$ and $T \in \mathcal{L}(X, Y)$ and $S \in \mathcal{L}(Y, Z)$. Let $\left(\alpha_{n}\right)$ and $\left(\beta_{n}\right)$ be sequences of ideal norms. Then $\alpha_{n} \prec \beta_{n}$ means that there exists a constant $c>0$ such that $\alpha_{n}(T) \leq c \beta_{n}(T)$ for all operators $T$ and $n=1,2, \ldots$ Moreover, $\alpha_{n} \asymp \beta_{n}$ is written for $\alpha_{n} \prec \beta_{n}$ and $\beta_{n} \prec \alpha_{n}$. Similar notation is used for sequences of real numbers.

Given three orthonormal systems $\mathcal{A}_{n}, \mathcal{B}_{n}$ and $\mathcal{C}_{n}$, the triangle inequality

$$
\varrho\left(\mathcal{C}_{n}, \mathcal{A}_{n}\right) \leq \varrho\left(\mathcal{C}_{n}, \mathcal{B}_{n}\right) \circ \varrho\left(\mathcal{B}_{n}, \mathcal{A}_{n}\right)
$$

is an immediate consequence of the definition. Furthermore, we have the general estimate

$$
\varrho\left(\mathcal{B}_{n}, \mathcal{A}_{n}\right) \leq \sqrt{n}
$$

We say that the orthonormal systems $\mathcal{A}_{n}=\left(a_{1}, \ldots, a_{n}\right) \subset L_{2}(M, \mu)$ and $\mathcal{B}_{n}=$ $\left(b_{1}, \ldots, b_{n}\right) \subset L_{2}(N, \nu)$ have identical distributions if for all Borel sets $\Omega \subset \mathbb{K}^{n}$

$$
\mu\left(\left\{s \in M:\left(a_{k}(s)\right) \in \Omega\right\}\right)=\nu\left(\left\{t \in N:\left(b_{k}(t)\right) \in \Omega\right\}\right) .
$$

Here $\mathbb{K}$ is the scalar field of either real or complex numbers. In this case

$$
\left(\int_{M}\left\|\sum_{k=1}^{n} a_{k}(t) x_{k}\right\|^{2} d \mu(s)\right)^{1 / 2}=\left(\int_{N}\left\|\sum_{k=1}^{n} b_{k}(s) x_{k}\right\|^{2} d \nu(t)\right)^{1 / 2}
$$

for all $x_{1}, \ldots, x_{n}$ in a Banach space. Hence, for any third orthonormal system $\mathcal{C}_{n}$,

$$
\varrho\left(\mathcal{A}_{n}, \mathcal{C}_{n}\right)=\varrho\left(\mathcal{B}_{n}, \mathcal{C}_{n}\right) \text { and } \varrho\left(\mathcal{C}_{n}, \mathcal{A}_{n}\right)=\varrho\left(\mathcal{C}_{n}, \mathcal{B}_{n}\right) \text {. }
$$

Let $\left(X_{k}\right)$ be a sequence of Banach spaces. We denote the $l_{2}$-sum of these spaces by $\left[l_{2}, X_{k}\right]$, i.e. $\left[l_{2}, X_{k}\right]$ consists of all sequences $x=\left(x_{k}\right)$ with $x_{k} \in X_{k}$ for which

$$
\left\|x \mid\left[l_{2}, X_{k}\right]\right\|=\left(\sum_{k}\left\|x_{k} \mid X_{k}\right\|^{2}\right)^{1 / 2}
$$

is finite. If $T_{k} \in \mathcal{L}\left(X_{k}, Y_{k}\right)$ for $k=1,2, \ldots$ with $\sup \left\|T_{k}\right\|<\infty$, we let $\left[l_{2}, T_{k}\right] \in$ $\mathcal{L}\left(\left[l_{2}, X_{k}\right],\left[l_{2}, Y_{k}\right]\right)$ be the diagonal operator that maps a sequence $\left(x_{k}\right)$ to the sequence $\left(T_{k} x_{k}\right) . \alpha$ is said to be $l_{2}$-stable if $\alpha\left(\left[l_{2}, T_{k}\right]\right)=\sup _{k} \alpha\left(T_{k}\right)$ for every sequence $T_{k} \in \mathcal{L}\left(X_{k}, Y_{k}\right)$ such that $\sup _{k} \alpha\left(T_{k}\right)<\infty$. The ideal norms $\varrho\left(\mathcal{B}_{n}, \mathcal{A}_{n}\right)$ are $l_{2}$-stable.

\section{Gaussian and Rademacher averages}

Let $\gamma_{n}$ be the canonical Gaussian measure on $\mathbb{R}^{n}$ defined by

$$
\gamma_{n}(B)=(2 \pi)^{-n / 2} \int_{B} \exp \left(-\frac{1}{2} \sum_{k=1}^{n} \sigma_{k}^{2}\right) d \sigma_{1} \ldots d \sigma_{n}
$$

for Borel sets $B \subset \mathbb{R}^{n}$. We simply write $\gamma$ for $\gamma_{1}$. We consider the Gaussian system $\mathcal{G}_{n}=\left(g_{1}, \ldots, g_{n}\right)$ as the system of coordinate functionals on $\left(\mathbb{R}^{n}, \gamma_{n}\right), g_{k}(s)=\sigma_{k}$ for $s=\left(\sigma_{1}, \ldots, \sigma_{n}\right) \in \mathbb{R}^{n}$. 
First of all, we provide some elementary facts about the distribution function

$$
D(u)=\gamma\{\sigma \in \mathbb{R}:|\sigma| \geq u\}=\sqrt{\frac{2}{\pi}} \int_{u}^{\infty} \exp \left(-\sigma^{2} / 2\right) d \sigma \text { for } u \geq 0 .
$$

$D$ is decreasing and $D(0)=1$. It follows from

$$
D(u)=\sqrt{\frac{2}{\pi}} \exp \left(-u^{2} / 2\right) \int_{0}^{\infty} \exp \left(-u \tau-\tau^{2}\right) d \tau
$$

that

$$
D(u) \leq \exp \left(-u^{2} / 2\right)
$$

Hence, for $q \geq 1$,

$$
\int_{0}^{\infty} D(u)^{1 / q} d u \leq \int_{0}^{\infty} \exp \left(-u^{2} / 2 q\right) d u=\sqrt{\frac{\pi q}{2}} .
$$

Moreover, it follows with the substitution

$$
v^{-1}=D(u) \text { and } v^{-2} d v=\sqrt{\frac{2}{\pi}} \exp \left(-u^{2} / 2\right) d u
$$

and (3.1) that

$$
\begin{aligned}
\int_{a}^{\infty} D(u)^{1 / 2} d u & =\sqrt{\frac{\pi}{2}} \int_{D(a)^{-1}}^{\infty} v^{-5 / 2} \exp \left(u^{2} / 2\right) d v \leq \sqrt{\frac{\pi}{2}} \int_{D(a)^{-1}}^{\infty} v^{-3 / 2} d v \\
& =\sqrt{2 \pi} D(a)^{1 / 2} .
\end{aligned}
$$

Integration by parts yields

$$
\begin{aligned}
\int_{u}^{\infty} \exp \left(-\sigma^{2} / 2\right) d \sigma & =\frac{1}{u} \exp \left(-u^{2} / 2\right)-\int_{u}^{\infty} \sigma^{-2} \exp \left(-\sigma^{2} / 2\right) d \sigma \\
& =\left(\frac{1}{u}-\frac{1}{u^{3}}\right) \exp \left(-u^{2} / 2\right)+3 \int_{u}^{\infty} \sigma^{-4} \exp \left(-\sigma^{2} / 2\right) d \sigma
\end{aligned}
$$

Consequently, we arrive at the classical estimate

$$
\left(\frac{1}{u}-\frac{1}{u^{3}}\right) \exp \left(-u^{2} / 2\right) \leq \sqrt{\frac{\pi}{2}} D(u) \leq \frac{1}{u} \exp \left(-u^{2} / 2\right) .
$$

Since

$$
\exp \left(1-u^{2}\right) \leq \sqrt{\frac{2}{\pi}}\left(\frac{1}{u}-\frac{1}{u^{3}}\right) \exp \left(-u^{2} / 2\right) \text { for } u \geq 3,
$$

we conclude that

$$
\sqrt{\frac{\pi}{2}} v^{-1} \exp \left(u^{2} / 2\right) \leq u^{-1} \leq(1+\log v)^{-1 / 2} .
$$

Defining $0=u_{1}<u_{2}<\ldots$ by $D\left(u_{m}\right)^{-1}=m$, we get

$$
u_{m} \geq(1+\log m)^{1 / 2}
$$


and

$$
\begin{aligned}
\int_{u_{m}}^{u_{m+1}} D(u)^{1 / 2} d u & =\sqrt{\frac{\pi}{2}} \int_{m}^{m+1} v^{-5 / 2} \exp \left(u^{2} / 2\right) d v \leq \int_{m}^{m+1} v^{-3 / 2}(1+\log v)^{-1 / 2} d v \\
& \leq m^{-3 / 2}(1+\log m)^{-1 / 2}
\end{aligned}
$$

for $m$ large enough to ensure $u_{m} \geq 3$. This proves that

$$
\int_{u_{m}}^{u_{m+1}} D(u)^{1 / 2} d u \prec m^{-3 / 2}(1+\log m)^{-1 / 2} .
$$

The next two lemmas lead to the inequalities between the ideal norms $\varrho\left(\mathcal{G}_{n}, \mathcal{R}_{n}\right)$ and $\varrho\left(\mathcal{I}_{n}, \mathcal{R}_{n}\right)$ in Theorem 3.3. The idea of the proof goes back to Kwapień; see [3], pp. 251-252.

Let $A$ be a measurable subset of $\mathbb{R}$ such that $\frac{1}{m+1}<\gamma(A) \leq \frac{1}{m}$ for some $m=1,2, \ldots$ We denote the indicator function of $A$ by $c_{A}$. Now we define functions $c_{1}^{A}, \ldots, c_{n}^{A}$ on $\mathbb{R}^{n}$ by

$$
c_{k}^{A}(s)=\gamma(A)^{-1 / 2} c_{A}\left(\sigma_{k}\right) \text { for } s=\left(\sigma_{1}, \ldots, \sigma_{n}\right) \text { and } k=1, \ldots, n .
$$

The system $\mathcal{C}_{n}^{A} \otimes \mathcal{R}_{n}=\left(c_{1}^{A} \otimes r_{1}, \ldots, c_{n}^{A} \otimes r_{n}\right)$ is orthonormal in $L_{2}\left(\mathbb{R}^{n} \times[0,1], \gamma_{n} \times \lambda\right)$, where $\lambda$ is the Lebesgue measure on $[0,1]$.

Lemma 3.1. With the above introduced notation, we have

$$
\varrho\left(\mathcal{C}_{n}^{A} \otimes \mathcal{R}_{n}, \mathcal{R}_{n}\right) \leq \sqrt{2} \varrho\left(\mathcal{I}_{m}, \mathcal{R}_{m}\right)
$$

Proof. We choose mutually disjoint subsets $A_{1}=A, A_{2}, \ldots, A_{m}$ in $\mathbb{R}$ such that $\gamma\left(A_{h}\right)=\gamma(A)$ for $h=1, \ldots, m$. Since the systems $\mathcal{C}_{n}^{A_{h}} \otimes \mathcal{R}_{n}$ have identical distributions, we conclude that

$$
\begin{aligned}
& \left\|\left(T x_{k}\right)\left|\mathcal{C}_{n}^{A} \otimes \mathcal{R}_{n}\left\|^{2}=\frac{1}{m} \sum_{h=1}^{m}\right\|\left(T x_{k}\right)\right| \mathcal{C}_{n}^{A_{h}} \otimes \mathcal{R}_{n}\right\|^{2} \\
& =\frac{1}{m} \int_{0}^{1} \int_{\mathbb{R}^{n}} \sum_{h=1}^{m}\left\|\sum_{k=1}^{n} T x_{k} c_{k}^{A_{h}}(s) r_{k}(t)\right\|^{2} d \gamma_{n}(s) d t \\
& \leq \frac{1}{m} \varrho\left(T \mid \mathcal{I}_{m}, \mathcal{R}_{m}\right)^{2} \int_{0}^{1} \int_{\mathbb{R}^{n}}^{1} \int_{0}^{1}\left\|\sum_{k=1}^{n} x_{k} r_{k}(t)\left(\sum_{h=1}^{m} c_{k}^{A_{h}}(s) r_{h}(u)\right)\right\|^{2} d u d \gamma_{n}(s) d t .
\end{aligned}
$$

We observe that

$$
\left|\sum_{h=1}^{m} c_{k}^{A_{h}}(s) r_{h}(u)\right| \leq \gamma(A)^{-1 / 2}
$$

since $A_{1}, \ldots, A_{m}$ are mutually disjoint. Then the contraction principle tells us that

$$
\int_{0}^{1}\left\|\sum_{k=1}^{n} x_{k} r_{k}(t)\left(\sum_{h=1}^{m} c_{k}^{A_{h}}(s) r_{h}(u)\right)\right\|^{2} d t \leq \gamma(A)^{-1}\left\|\left(x_{k}\right) \mid \mathcal{R}_{n}\right\|^{2} .
$$

Hence

$$
\left\|\left(T x_{k}\right)\left|\mathcal{C}_{n}^{A} \otimes \mathcal{R}_{n}\left\|\leq(m \gamma(A))^{-1 / 2} \varrho\left(T \mid \mathcal{I}_{m}, \mathcal{R}_{m}\right)\right\|\left(x_{k}\right)\right| \mathcal{R}_{n}\right\|
$$


Finally, $\frac{1}{m+1}<\gamma(A) \leq \frac{1}{m}$ implies that

$$
\frac{1}{m} \leq \frac{2}{m+1}<2 \gamma(A)
$$

So we get

$$
\left\|\left(T x_{k}\right)\left|\mathcal{C}_{n}^{A} \otimes \mathcal{R}_{n}\left\|\leq \sqrt{2} \varrho\left(T \mid \mathcal{I}_{m}, \mathcal{R}_{m}\right)\right\|\left(x_{k}\right)\right| \mathcal{R}_{n}\right\| .
$$

A look at the definition of $\varrho\left(T \mid \mathcal{C}_{n}^{A} \otimes \mathcal{R}_{n}, \mathcal{R}_{n}\right)$ finishes the proof.

Lemma 3.2. $\varrho\left(\mathcal{G}_{n}, \mathcal{R}_{n}\right) \leq \sqrt{2} \sum_{m=1}^{\infty} \int_{u_{m}}^{u_{m+1}} \sqrt{D(u)} d u \varrho\left(\mathcal{I}_{m}, \mathcal{R}_{m}\right)$.

Proof. We let $A_{u}=\{\sigma \in \mathbb{R}:|\sigma| \geq u\}$ for $u \geq 0$ and note that $D(u)=\gamma\left(A_{u}\right)$. Now we observe that

$$
\left|g_{k}(s)\right|=\left|\sigma_{k}\right|=\int_{0}^{\infty} \sqrt{\gamma\left(A_{u}\right)} c_{k}^{A_{u}}(s) d u \text { for } s=\left(\sigma_{1}, \ldots, \sigma_{n}\right) \in \mathbb{R}^{n} \text { and } k=1, \ldots, n \text {. }
$$

Letting $\left|\mathcal{G}_{n}\right| \otimes \mathcal{R}_{n}=\left(\left|g_{1}\right| \otimes r_{1}, \ldots,\left|g_{n}\right| \otimes r_{n}\right)$, it follows from Lemma 3.1 that

$$
\begin{aligned}
\left\|\left(T x_{k}\right)\right\| \mathcal{G}_{n} \mid \otimes \mathcal{R}_{n} \| & \leq \int_{0}^{\infty} \sqrt{\gamma\left(A_{u}\right)}\left\|\left(T x_{k}\right) \mid \mathcal{C}_{n}^{A_{u}} \otimes \mathcal{R}_{n}\right\| d u \\
& \leq \sqrt{2} \sum_{m=1}^{\infty} \int_{u_{m}}^{u_{m+1}} \sqrt{\gamma\left(A_{u}\right)} d u \varrho\left(T \mid \mathcal{I}_{m}, \mathcal{R}_{m}\right)\left\|\left(x_{k}\right) \mid \mathcal{R}_{n}\right\| .
\end{aligned}
$$

The observation that the systems $\left|\mathcal{G}_{n}\right| \otimes \mathcal{R}_{n}$ and $\mathcal{G}_{n}$ have identical distributions finishes the proof.

We now come to the announced inequalities.

\section{Theorem 3.3.}

$$
\max _{1 \leq m \leq n}\left(\frac{1+\log m}{m}\right)^{1 / 2} \varrho\left(\mathcal{I}_{m}, \mathcal{R}_{m}\right) \prec \varrho\left(\mathcal{G}_{n}, \mathcal{R}_{n}\right) \prec \sum_{m=1}^{n} m^{-3 / 2}(1+\log m)^{-1 / 2} \varrho\left(\mathcal{I}_{m}, \mathcal{R}_{m}\right) .
$$

Moreover, there exists a constant $c \geq 1$ such that for $2 \leq q<\infty$

$$
\varrho\left(\mathcal{G}_{n}, \mathcal{R}_{n}\right) \leq c \sqrt{q} \varrho_{2}^{(q)}\left(\mathcal{I}_{n}, \mathcal{R}_{n}\right) .
$$

Proof. Let $M$ be an $n$-dimensional subspace of $X$ and denote the inclusion map of $M$ in $X$ by $J_{M}^{X}$. John's theorem (see [6], p. 54) tells us that $d\left(M, l_{2}^{n}\right) \leq \sqrt{n}$. Hence

$$
\varrho\left(T J_{M}^{X} \mid \mathcal{I}_{m}, \mathcal{R}_{m}\right) \leq\|T\| d\left(M, l_{2}^{n}\right) \varrho\left(l_{2}^{n} \mid \mathcal{I}_{m}, \mathcal{R}_{m}\right) \leq \sqrt{n}\|T\|
$$


for $m=1,2, \ldots$ Then it follows from Lemma 3.2 and (3.3) that

$$
\begin{aligned}
& \varrho\left(T J_{M}^{X} \mid \mathcal{G}_{n}, \mathcal{R}_{n}\right) \leq \sqrt{2} \sum_{m=1}^{n} \int_{u_{m}}^{u_{m+1}} D(u)^{1 / 2} d u \varrho\left(T J_{M}^{X} \mid \mathcal{I}_{m}, \mathcal{R}_{m}\right) \\
& \quad+\sqrt{2} \sum_{m=n+1}^{\infty} \int_{u_{m}}^{u_{m+1}} D(u)^{1 / 2} d u \varrho\left(T J_{M}^{X} \mid \mathcal{I}_{m}, \mathcal{R}_{m}\right) \\
& \leq \sqrt{2} \sum_{m=1}^{n} \int_{u_{m}}^{u_{m+1}} D(u)^{1 / 2} d u \varrho\left(T \mid \mathcal{I}_{m}, \mathcal{R}_{m}\right)+\sqrt{2 n} \int_{u_{n+1}}^{\infty} D(u)^{1 / 2} d u\|T\| \\
& \leq \sqrt{2} \sum_{m=1}^{n} \int_{u_{m}}^{u_{m+1}} D(u)^{1 / 2} d u \varrho\left(T \mid \mathcal{I}_{m}, \mathcal{R}_{m}\right)+2 \sqrt{\pi}\|T\| .
\end{aligned}
$$

Since

$$
\varrho\left(T \mid \mathcal{G}_{n}, \mathcal{R}_{n}\right)=\sup \left\{\varrho\left(T J_{M}^{X} \mid \mathcal{G}_{n}, \mathcal{R}_{n}\right): M n \text {-dimensional subspace of } X\right\},
$$

we obtain

$$
\varrho\left(T \mid \mathcal{G}_{n}, \mathcal{R}_{n}\right) \prec \sum_{m=1}^{n} \int_{u_{m}}^{u_{m+1}} D(u)^{1 / 2} d u \varrho\left(T \mid \mathcal{I}_{m}, \mathcal{R}_{m}\right) .
$$

Then the upper estimate in the first part of the assertion is a consequence of inequality (3.5). The second assertion can be seen as follows. For $q \geq 2$, we conclude from Hölder's inequality that

$$
\varrho\left(\mathcal{I}_{m}, \mathcal{R}_{m}\right) \leq m^{1 / 2-1 / q} \varrho_{2}^{(q)}\left(\mathcal{I}_{m}, \mathcal{R}_{m}\right) .
$$

Moreover,

$$
m^{1 / 2-1 / q} \leq D\left(u_{m}\right)^{1 / q-1 / 2} \leq D(u)^{1 / q-1 / 2} \text { for } u \geq u_{m} .
$$

By (3.2) and (3.6),

$$
\varrho\left(\mathcal{G}_{n}, \mathcal{R}_{n}\right) \prec \int_{0}^{u_{n+1}} D(u)^{1 / q} d u \varrho_{2}^{(q)}\left(\mathcal{I}_{n}, \mathcal{R}_{n}\right) \prec \sqrt{q} \varrho_{2}^{(q)}\left(\mathcal{I}_{n}, \mathcal{R}_{n}\right) .
$$

Finally, we make use of the estimate

$$
\varrho\left(\mathcal{I}_{m}, \mathcal{G}_{m}\right) \prec \frac{m^{1 / 2}}{(1+\log m)^{1 / 2}}
$$

which we state without proof. This well-known fact can be found in [5]. Then the triangle inequality implies

$$
\varrho\left(\mathcal{I}_{m}, \mathcal{R}_{m}\right) \leq \varrho\left(\mathcal{I}_{m}, \mathcal{G}_{m}\right) \circ \varrho\left(\mathcal{G}_{m}, \mathcal{R}_{m}\right) \prec \frac{m^{1 / 2}}{(1+\log m)^{1 / 2}} \varrho\left(\mathcal{G}_{n}, \mathcal{R}_{n}\right)
$$

which proves the lower estimate.

Remark 3.4. The factor $\sqrt{q}$ in the second inequality of the preceding theorem gives the right order of magnitude for $q \rightarrow \infty$. In fact, using the identity of $l_{\infty}^{n}$ for some $n$ satisfying $e^{q-1} \leq n \leq e^{q}$, we conclude from Example 4.4 that

$$
\varrho\left(l_{\infty}^{n} \mid \mathcal{G}_{n}, \mathcal{R}_{n}\right) \asymp(1+\log n)^{1 / 2} \geq \sqrt{q} \text { and } \varrho_{2}^{(q)}\left(l_{\infty}^{n} \mid \mathcal{I}_{n}, \mathcal{R}_{n}\right)=n^{1 / q} \leq e .
$$


The lower and upper estimates in the first assertion of the theorem are quite close as will be also indicated in the next section. However, in all checked examples actually the lower estimate is attained up to a constant. So the question arises whether the Rademacher cotype ideal norms exactly determine the behaviour of the sequence $\varrho\left(\mathcal{G}_{n}, \mathcal{R}_{n}\right)$. More precisely, do we even have

$$
\varrho\left(\mathcal{G}_{n}, \mathcal{R}_{n}\right) \asymp \max _{1 \leq m \leq n} \frac{(1+\log m)^{1 / 2}}{m^{1 / 2}} \varrho\left(\mathcal{I}_{m}, \mathcal{R}_{m}\right) ?
$$

\section{Applications}

It is well-known that

$$
\varrho\left(\mathcal{G}_{n}, \mathcal{R}_{n}\right) \prec \sqrt{1+\log n} .
$$

This also follows from Theorem 3.3. Indeed, we conclude from the right hand inequality in this theorem that

$$
\begin{aligned}
\varrho\left(\mathcal{G}_{n}, \mathcal{R}_{n}\right) & \prec \sum_{m=1}^{n} m^{-3 / 2}(1+\log m)^{-1 / 2} \varrho\left(\mathcal{I}_{m}, \mathcal{R}_{m}\right) \leq \sum_{m=1}^{n} m^{-1}(1+\log m)^{-1 / 2} \\
& \asymp(1+\log n)^{1 / 2} .
\end{aligned}
$$

For a convenient formulation of the main result of this paper let us introduce the following operator ideals. For $2 \leq q<\infty$, let $\mathcal{R} C_{q}$ denote the ideal of Rademacher cotype $q$ operators, that is, $T \in \mathcal{R} C_{q}$ if the sequence $\varrho_{2}^{(q)}\left(T \mid \mathcal{I}_{n}, \mathcal{R}_{n}\right)$ is bounded. We say that $T$ has Rademacher subcotype if $\varrho\left(T \mid \mathcal{I}_{n}, \mathcal{R}_{n}\right)=o(\sqrt{n})$. The ideal of Rademacher subcotype operators is denoted by $\mathcal{R} C$. Finally, let $\mathcal{G} R$ and $\mathcal{Q} G R$ be the ideals of all operators $T$ satisfying

$$
\varrho\left(T \mid \mathcal{G}_{n}, \mathcal{R}_{n}\right)=O(1) \text { and } \varrho\left(T \mid \mathcal{G}_{n}, \mathcal{R}_{n}\right)=o(\sqrt{1+\log n}) \text {, respectively. }
$$

Theorem 4.1. $\bigcup_{2 \leq q<\infty} \mathcal{R} C_{q} \subset \mathcal{G} R \subset \mathcal{Q} G R=\mathcal{R} C$.

Proof. The second assertion in Theorem 3.3 implies that

$$
\bigcup_{2 \leq q<\infty} \mathcal{R} C_{q} \subset \mathcal{G} R
$$

$\mathcal{G} R \subset \mathcal{Q} G R$ is trivial. The lower estimate in the first assertion in Theorem 3.3 yields $\mathcal{Q} G R \subset \mathcal{R} C$. Finally, the reverse inclusion is a consequence of the upper estimate there. Indeed, given $T \in \mathcal{R} C$ and $\varepsilon>0$, we successively choose $m_{0}$ and $n_{0}$ such that

$$
\varrho\left(T \mid \mathcal{I}_{m}, \mathcal{R}_{m}\right) \leq \varepsilon m^{1 / 2} \text { for } m \geq m_{0} \text { and } \frac{1+\log m_{0}}{1+\log n_{0}} \leq \varepsilon^{2} .
$$


Then we get for $n \geq n_{0}$ that

$$
\begin{aligned}
& \varrho\left(T \mid \mathcal{G}_{n}, \mathcal{R}_{n}\right) \prec \sum_{m=1}^{m_{0}} m^{-3 / 2}(1+\log m)^{-1 / 2} \varrho\left(T \mid \mathcal{I}_{m}, \mathcal{R}_{m}\right) \\
& +\sum_{m=m_{0}+1}^{n} m^{-3 / 2}(1+\log m)^{-1 / 2} \varrho\left(T \mid \mathcal{I}_{m}, \mathcal{R}_{m}\right) \\
& \leq \sum_{m=1}^{m_{0}} m^{-1}(1+\log m)^{-1 / 2}\|T\|+\varepsilon \sum_{m=m_{0}+1}^{n} m^{-1}(1+\log m)^{-1 / 2} \\
& \prec\left(1+\log m_{0}\right)^{1 / 2}\|T\|+\varepsilon(1+\log n)^{1 / 2} \leq \varepsilon(\|T\|+1)(1+\log n)^{1 / 2} .
\end{aligned}
$$

Remark 4.2. Note that Theorem 4.1 implies the dichotomy due to Maurey and Pisier mentioned in the introduction. Indeed, it is well-known from [4] that, for a Banach space $X, I d_{X} \in \mathcal{R} C$ implies $I d_{X} \in \mathcal{R} C_{q}$ for some $q<\infty$. We also refer to [1] for an elementary proof of this fact. Hence we get from Theorem 4.1

$$
I d_{X} \in \mathcal{R} C \Longleftrightarrow I d_{X} \in \mathcal{Q} G R \Longleftrightarrow I d_{X} \in \mathcal{G} R \Longleftrightarrow I d_{X} \in \mathcal{R} C_{q} \text { for some } q<\infty .
$$

Moreover, these conditions are equivalent to the condition that $X$ does not contain the spaces $l_{\infty}^{n}$ uniformly.

We now prepare examples of diagonal operators in $\left[l_{2}, l_{\infty}^{2^{k}}\right]$ showing that the inclusions in Theorem 4.1 are strict. The following examples should be well-known. For a proof of the first one, we refer the reader to [5].

Example 4.3. $\varrho\left(l_{\infty}^{N} \mid \mathcal{I}_{n}, \mathcal{R}_{n}\right)=\min \{\sqrt{N}, \sqrt{n}\}$.

Example 4.4. $\varrho\left(l_{\infty}^{N} \mid \mathcal{G}_{n}, \mathcal{R}_{n}\right) \asymp \min \{\sqrt{1+\log N}, \sqrt{1+\log n}\}$.

Proof. Let $M=\min \{N, n\}$. It follows from Theorem 3.3 and Example 4.3 that

$$
\begin{aligned}
\varrho\left(l_{\infty}^{N} \mid \mathcal{G}_{n}, \mathcal{R}_{n}\right) & \prec \sum_{m=1}^{n} m^{-3 / 2}(1+\log m)^{-1 / 2} \varrho\left(l_{\infty}^{N} \mid \mathcal{I}_{m}, \mathcal{R}_{m}\right) \\
& =\sum_{m=1}^{n} m^{-3 / 2}(1+\log m)^{-1 / 2} \min \{\sqrt{N}, \sqrt{m}\} \\
& \leq \sum_{m=1}^{M} m^{-1}(1+\log m)^{-1 / 2}+\sqrt{N} \sum_{m=N+1}^{\infty} m^{-3 / 2} \\
& \prec \sqrt{1+\log M} .
\end{aligned}
$$

For the reverse estimate, let $u_{1}, \ldots, u_{N}$ be the unit vector basis of $l_{\infty}^{N}$ and observe that $\left\|\left(u_{k}\right) \mid \mathcal{R}_{M}\right\|=1$. Since

$$
\begin{aligned}
\left\|\left(u_{k}\right) \mid \mathcal{G}_{M}\right\| & =\left(\int_{\mathbb{R}^{M}}\left\|\sum_{k=1}^{M} g_{k}^{(M)}(s) u_{k}\right\|^{2} d \gamma_{M}(s)\right)^{1 / 2} \\
& =\left(\int_{\mathbb{R}^{M}} \max _{1 \leq k \leq M}\left|g_{k}^{(M)}(s)\right|^{2} d \gamma_{M}(s)\right)^{1 / 2}
\end{aligned}
$$


we conclude that

$$
\varrho\left(l_{\infty}^{N} \mid \mathcal{G}_{n}, \mathcal{R}_{n}\right) \geq \varrho\left(l_{\infty}^{M} \mid \mathcal{G}_{M}, \mathcal{R}_{M}\right) \geq\left(\int_{\mathbb{R}^{M}} \max _{1 \leq k \leq M}\left|g_{k}^{(M)}(s)\right|^{2} d \gamma_{M}(s)\right)^{1 / 2}
$$

Finally, we have

$$
\left(\int_{\mathbb{R}^{M}} \max _{1 \leq k \leq M}\left|g_{k}^{(M)}(s)\right|^{2} d \gamma_{M}(s)\right)^{1 / 2} \asymp \sqrt{1+\log M}
$$

see $[3]$, p. 80.

Now the following example shows that the inclusions in Theorem 4.1 are strict.

Example 4.5. For $0 \leq \alpha \leq 1 / 2$, we consider the diagonal operator

$$
\begin{aligned}
D_{\alpha}:\left[l_{2}, l_{\infty}^{2^{k}}\right] & \longrightarrow\left[l_{2}, l_{\infty}^{2^{k}}\right] \\
\left(x_{k}\right) & \longmapsto\left(k^{-\alpha} x_{k}\right) .
\end{aligned}
$$

Then

$$
D_{1 / 2} \in \mathcal{G} R \backslash \bigcup_{2<q<\infty} \mathcal{R} C_{q} \text { and } D_{\alpha} \in \mathcal{Q} G R \backslash \mathcal{G} R \text { for } 0<\alpha<1 / 2
$$

Proof. It follows from the $l_{2}$-stability of $\varrho\left(\mathcal{G}_{n}, \mathcal{R}_{n}\right)$ and Example 4.4 that

$$
\begin{aligned}
\varrho\left(D_{\alpha} \mid \mathcal{G}_{n}, \mathcal{R}_{n}\right) & =\sup _{k} k^{-\alpha} \varrho\left(l_{\infty}^{2^{k}} \mid \mathcal{G}_{n}, \mathcal{R}_{n}\right) \asymp \sup _{k} k^{-\alpha} \min \{\sqrt{1+\log n}, \sqrt{k}\} \\
& =(1+\log n)^{1 / 2-\alpha} .
\end{aligned}
$$

Hence

$$
D_{1 / 2} \in \mathcal{G} R \text { and } D_{\alpha} \in \mathcal{Q} G R \backslash \mathcal{G} R \text { for } 0<\alpha<1 / 2 \text {. }
$$

Furthermore, the $l_{2}$-stability of $\varrho\left(\mathcal{I}_{n}, \mathcal{R}_{n}\right)$ and Example 4.3 imply that

$$
\begin{aligned}
\varrho\left(D_{\alpha} \mid \mathcal{I}_{n}, \mathcal{R}_{n}\right) & =\sup _{k} k^{-\alpha} \varrho\left(l_{\infty}^{2^{k}} \mid \mathcal{I}_{n}, \mathcal{R}_{n}\right) \\
& =\sup _{k} k^{-\alpha} \min \left\{\sqrt{n}, 2^{k / 2}\right\} \asymp \sqrt{n}(1+\log n)^{-\alpha} .
\end{aligned}
$$

Then we conclude from Hölder's inequality that

$$
\varrho_{2}^{(q)}\left(D_{\alpha} \mid \mathcal{I}_{n}, \mathcal{R}_{n}\right) \geq n^{1 / q-1 / 2} \varrho\left(D_{\alpha} \mid \mathcal{I}_{n}, \mathcal{R}_{n}\right) \asymp n^{1 / q}(1+\log n)^{-\alpha} .
$$

This shows that $D_{\alpha} \notin \mathcal{R} C_{q}$ for any $q<\infty$.

\section{REFERENCES}

1. A. Hinrichs, On the type constants with respect to systems of characters of a compact abelian group, Stud. Math. 118 (1996), 231-243. MR 97i:46022

2. S. Kwapień, Isomorphic characterizations of inner product spaces by orthogonal series with vector valued coefficients, Stud. Math. 44 (1972), 583-595. MR 49:5789

3. M. Ledoux and M. Talagrand, Probability in Banach spaces, Springer, Berlin, Heidelberg, New York, 1991. MR 93c:60001 
4. B. Maurey and G. Pisier, Séries de variables aléatoires vectorielles indépendantes et propriétés géométriques des espaces de Banach, Stud. Math. 58 (1976), 45-90. MR 56:1388

5. A. Pietsch and J. Wenzel, Orthonormal systems and Banach space geometry, Cambridge Univ. Press, 1998. CMP 99:01

6. N. Tomczak-Jaegermann, Banach-Mazur distances and finite-dimensional operator ideals, Longman, 1989. MR 90k:46039

Mathematical Institute, Friedrich-Schiller-University, D-07743 Jena, Germany

E-mail address: nah@rz.uni-jena.de 